\title{
DNA nanotechnology for building artificial dynamic systems - ERRATUM
}

doi: $10.1557 / \mathrm{mrs} .2019 .155$.

In the original publication of the article titled "DNA nanotechnology for building artificial dynamic systems," an incorrect image was used as the graphical abstract and the title page image. The Materials Research Society apologizes for this discrepancy. The online version of the article has been corrected appropriately.

\section{Reference}

1. N. Liu, MRS Bull. 44 (7), 576 (2019). doi: 10.1557/mrs.2019.155. 